\title{
Harmonic detection by using different artificial neural network topologies
}

\author{
J.L. Flores Garrido y P. Salmerón Revuelta \\ Department of Electrical Engineering \\ E. P. S., Huelva University \\ Ctra de Palos de la Frontera s/n 21819, Palos de la Frontera. Huelva (Spain) \\ phone:+34 959 017583, fax:+34 959017304 \\ e-mail: : juan.flores@dfaie.uhu.es, patricio@uhu.es
}

\begin{abstract}
At this paper the performance of different artificial neural networks (ANN) topologies has been analized for harmonic detection by distorted waveforms. With this information, it's possible to obtain the reference signal for an active power filter (APF) control by nonlinear loads compensation. In particular, two ANN types, the static multilayer perceptron (MLP) and the dynamic MLP, stand out as the most suitable for distorsion identifying. Acceptable results were also obtained with recurrent networks, but with a lower performance than with the other topologies. Two different control strategies have been applied. One of them is based in the static MLP, neural network that has proved to be the most appropiate by measuring the rectangular components of the signal harmonics. The other strategy, based in the dynamic MLP, permits extracting the instantaneous value of the fundamental waveform. The three mentioned ANN topologies have been conveniently trained and simulated with waveforms distorted by several harmonics. Finally, the obtained results with practical cases of harmonic distorted waveforms are presented and discussed.
\end{abstract}

\section{Key words}

Harmonics, Artificial neural network, Active power filter, Electric power quality.

\section{Introduction}

In the last years a high increase of problems in the electric power distribution networks due to the presence of harmonics has been observed. At the moment, one of the most important tools for correcting the lack of electric power quality are the active power filters (APF), that, thanks to the recent development of signal processing and power converters, are a growing reality, [1],[5].

The objective of this work has been to find the most suitable design strategies for use of the ANNs by the APFs. In this way, different network topologies have been analyzed, following two different strategies or working methods for harmonic distortion detection. The diagram in Fig. 1 shows the application of the neural network to an ANN-controlled APF (Fig. 1). [5],[6].

The used neural networks are of those with off-line training, so that the performance times are shorter than in another systems such as the application of adaptative techniques.

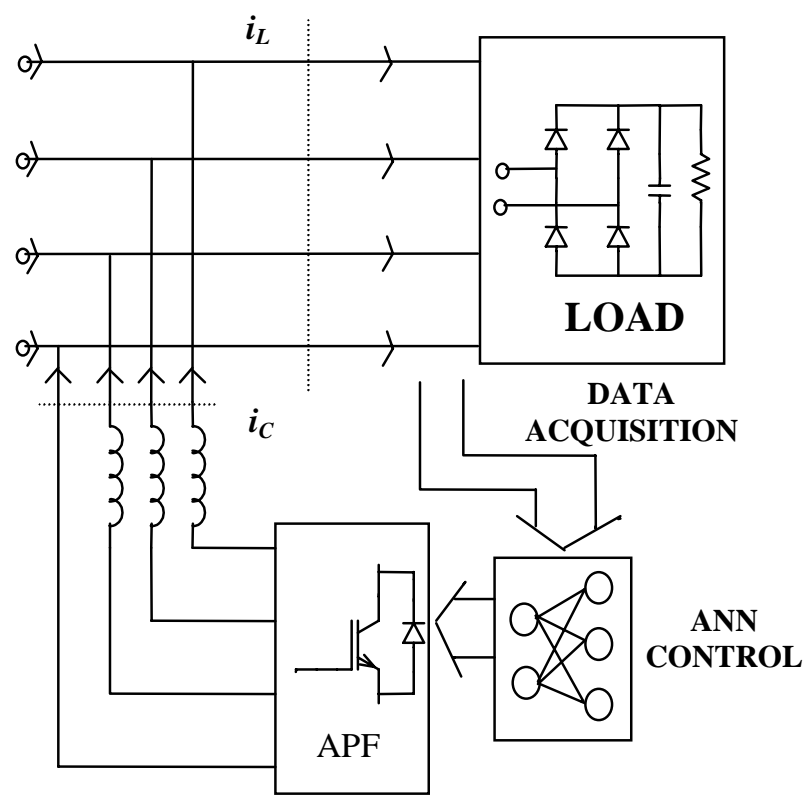

Fig. 1. ANN-controlled APF

The distortion current is used in the APF as the reference signal for controlling of the power stage. The two different strategies, from the point of view of the way in which the distortion current is obtained, are the following:

1) Obtaining the content of harmonics present in the current signal, expressed as in the Fourier series expansion. By each period of the acquired signal, the ANN outputs the rectangular components coefficients of the more relevant harmonics. With this set of coefficients, the distortion current can be generated and used for controlling of the power converter (Fig. 2).

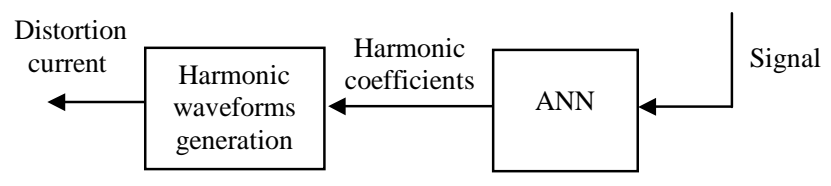

Fig. 2. Harmonic coefficients strategy 
2) In the second strategy, the acquired signal is sequentialy introduced in the ANN, and as a result, this network outputs the fundamental waveform in real time. The distortion current is hence obtained as the difference between the original signal and its fundamental component, Fig. 3.

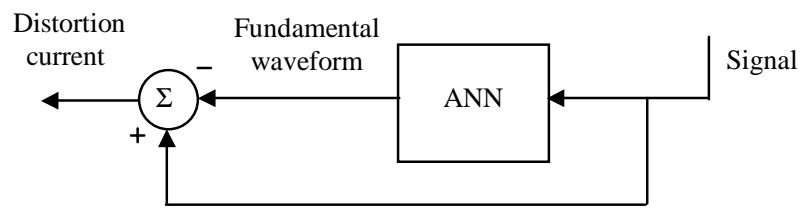

Fig. 3. Fundamental waveform strategy

\section{Artificial neural networks for harmonic detection}

\section{A. Neural Networks}

A neural network is an interconnection of processing units (artificial neurons). These neurons consists of three components, as can be seen in Fig. 4: the weighted connections, the combination function and the transfer function. The input signals flow through the connections and then accumulate in the summing node. The connection weights can be positive or negative. By means of these weights, the connection modulates the amount of information passing between to the summing node. The most common combination function is an addition. The summed signal, $n$, flows to the output through a transfer function, $f$. These function can be linear or nonlinear type. Among the nonlinear ones the most frequently used are sigmoidal. This transfer function produces the neuron output.

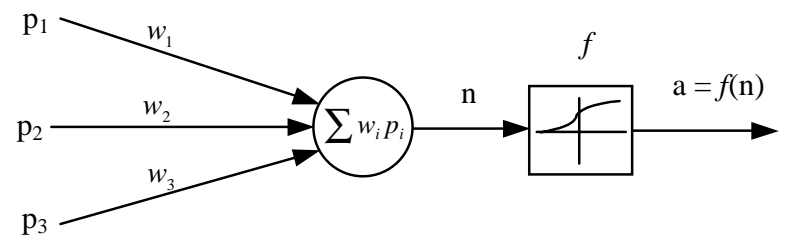

Fig. 4. Components of an artificial neuron

The transfer functions of the ANNs applied in this work are shown in Table I. The first function is linear. The other two are sigmoidal nonlinear functions, and are by neural networks commonly employed to give then a nonlinear feature. In all topologies of this work a combination of linear and nonlinear functions is used.

Neural networks are organized into layers of neurons. All neurons in a layer have the same inputs and the same trasnfer function. There is also the so called "input layer", formed by input units, that are not neurons, but constitute one more component in the ANN topology. The different layers of a typical ANN can be seen in Fig. 5. There can be one or more hidden layers. The number of layers and the number of neurons in each layer depend on the complexity of the problem being solved and the desired accuracy.

TABLE I. Employed transfer functions

\begin{tabular}{|c|c|}
\hline Linear & $a=n$ \\
\hline Log-Sigmoid & $a=\frac{1}{1+e^{-n}}$ \\
\hline Tan-Sigmoid & $a=\frac{2}{1+e^{-2 n}}-1$ \\
\hline
\end{tabular}

\section{B. Selected topologies}

There are many different ANN topologies. A classification can be made depending on the existence of feedback in the network. In the so called recurrent networks the interconnections of the neurons make possible feedback of the data flow between layers. With this, a certain "shortterm memory" is achieved, since the information introduced into the network temporarily influences the later response of their neurons. The Elman network belongs to this group, and another examples are Jordan, Kohonen or Hopfield networks, [2],[3].

By the networks without feedback (feedforward networks), the information always flows fordwards. So the inputs for the elements of a layer can only come from the ouputs of the preceding neurons in the direction of data flow. The main ANN type at this group is the Multilayer Perceptron network.

The perceptron network contains neurons with nonlinear functions, what gives the network the ability to emulate nonlinear mapping properties, and it makes the network suitable for many applications, without the limitations of pure linear networks. Furthermore, it is proved (Kolmogorov's Theorem) that a two neuron-layer perceptron network with nonlinear function can generate any function as output with the desired approximation. This all, together with the results obtained by other authors at the application to sinusoidal waveforms [5]-[7],[10], was the reason for selecting this topology for the purpose of harmonic detection in distorted waveforms. In particular, the MLP has shown to be an appropiate network for the first strategy. A set of waveform values corresponding to a period is introduced, obtaining at the output the rectangular components of the harmonics of interest. The topology of this ANN can be seen at the Fig. 5. Here appears a simplified network consisted of 3 input units, 3 neurons in the hidden layer and 2 neurons in the output layer.

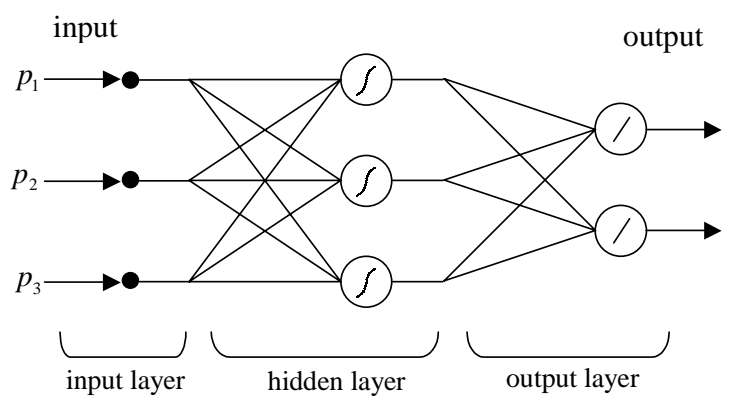

Fig. 5. Multilayer Perceptron ANN 
The transfer functions are tan-sigmoid (with values between -1 and 1) or log-sigmoid (with values between 0 and 1) in the hidden layer, and linear in the output layer. The applied learning algorithm is the Backpropagation.

For the second working method applied to the detection of the harmonic distortion, by which the fundamental harmonic is extracted in a dynamic way, by introducing the signal data sequentially, networks are proposed, that have the property of the "shortterm memory", so being able to mantain temporarily information of the former input data, as is the case of the recurrent networks. The Elman network has been taken as a typical recurrent ANN, very often applied to problems with common characteristics with the purpose of this work. This network consists of two neuron layers and has internal feedback from the outputs of the hidden layer to the input layer, so being these signals again introduced as inputs for the neurons of the hidden layer. The employed transfer functions are in both layers as in the case of the MLP already described. As learning algorithm for the training of the network it will be used again the Backpropagation. Fig. 6 shows this topology, with 2 inputs, 3 neurons in the hidden layer and 2 neurons in the output layer.

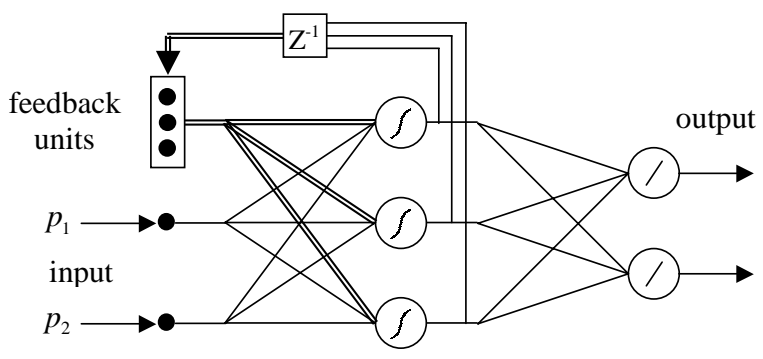

Fig. 6. Elman recurrent network

And another topology that owns the efectiveness of the MLP networks, as also the dynamic features of the recurrent networks, in the way that it mantains memory of the last values of the input signal, is the Dynamic Multilayer Perceptron. Good results are being achieved with this type of ANN in a lot of applications $[4],[5],[8],[9]$. At this topology the input layer to the neural network is a time delayed series of input signal values. The first input unit receives the instantaneous value of the signal, sequentially introduced. The other input units have the delayed values of the input signal. These set of time delayed input values can be observed in Fig. 7.

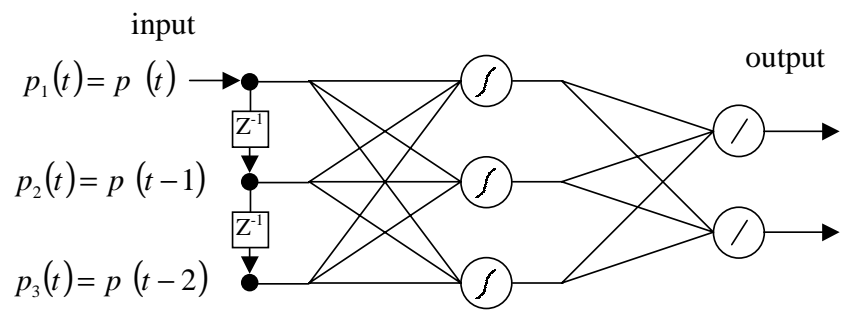

Fig. 7. Dynamic MLP network

\section{Harmonic detection by using Multilayer Perceptron.}

The different described neural networks have been trained and simulated in many different ways with the goal of getting the parameter values that produce the optimal performance for each of the topologies. Two different performance indices were chosen to evaluate the efectiveness of each studied network by both of the operation strategies: the reached accuracy level (in terms of the error rate) and the required training time. With respect to the accuracy level, at the measuring of the harmonic coefficients an error rate of $1 \%$ were estimated as minimum desired accuracy. With respect to the training time, network topologies and parameters were desired, that could be trained in so short times as possible. For this purpose it were compared the needed times to achieve similar accuracy levels.

The software tool employed has been the Neural Networks Toolbox of Matlab. All types of available training algorithms were used and tested, and the most efficient was found to be the Levenberg-Marquardt modified Backpropagation.

\section{A. Approach to the problem and parameter adjustments}

The detection of the parameters that define a sinusoidal waveform (the amplitude and the phase angle) is a good previous step for testing the capabilities of this ANN at the more general task of obtaining the distortion harmonics coefficients. At this first stage an approach to the more important parameters is made. These are the number and type of the needed training pairs, and the suitable number of neurons, among others. The general equation of a sinusoidal voltage or current waveform to the angular frequency $\omega$ is as follows:

$$
x(t)=X \cos (\omega t+\phi)
$$

where $X$ is the amplitude and $\phi$ is the initial phase angle. This equation can be substituted with the following expression, more appropiate for the desired working method to apply:

$$
x(t)=A \cos \omega t+B \operatorname{sen} \omega t
$$

So the objective is to detect the rectangular components $\mathrm{A}$ and $\mathrm{B}$ of the wave. For this, the network must be trained with a set of training pairs that are representative enough of all possible sinusoidal waveforms. At this first stage the work deals particularly with what could be a voltage waveform, with amplitudes between 210 and 230 $\mathrm{V}_{\mathrm{RMS}}$, and phase angles between $-90^{\circ}$ and $90^{\circ}$. The more suitable sampling frequency was found to be of 32 points per period. This showed to be valid for all proofs and network topologies used at this work.

The set of training pairs was formed by a number of waveforms with different amplitudes and phase angles within the described ranges. The minimum number of necessary training pairs for the networks to learn with enough accuracy was 20 . And as regards the number of 
hidden layers of neurons, better results were obtained with a single layer as with two ones. The optimal number of neurons was 8 . In Table II some results are shown with different numbers of neurons within the range from 4 to 12. For this proof a minimum accuracy was required, measured by means of the mean square error (mse) of the network outputs for the full set of training example waves comparing with the exact values of the training pairs. It was established an error mse $=1 \mathrm{E}-10$ as goal. The first column in the table shows the reached accuracy, and in the other two columns it can be seen the time it required in terms of seconds and number of iterations, executed in a PC Pentium 1.7 GHz and 256 MB RAM.

TABLE II. Optimization of the number of neurons

\begin{tabular}{|c|c|c|c|}
\hline $\begin{array}{c}\text { number of } \\
\text { neurons }\end{array}$ & mse & time (s) & $\begin{array}{c}\text { number of } \\
\text { iterations }\end{array}$ \\
\hline 4 & $9.9 \mathrm{e}-10$ & 42 & 998 \\
\hline 6 & $7.3 \mathrm{e}-10$ & 4.4 & 35 \\
\hline 7 & $5.3 \mathrm{e}-10$ & 4.1 & 24 \\
\hline 8 & $1.1 \mathrm{e}-12$ & 3.8 & 16 \\
\hline 10 & $6.1 \mathrm{e}-10$ & 5.2 & 10 \\
\hline 12 & $7.9 \mathrm{e}-10$ & 10.8 & 13 \\
\hline
\end{tabular}

Next the generalization for inputs different of those used as training pairs were proved. The proofs are made with the $8+2$ neurons network. 8 neurons in the hidden layer and 2 neurons in the output layer (for the two coefficients). The input layer has 32 units in all simulations. This was the minimum number of data per period necessary for supplying the network enough information of the wave. With 64 inputs the times are longer and no improvement could be seen, and that was so with all other ANNs and proofs in this work. After the training with 20 different waveforms, all error rates with other different inputs were around $0.01 \%$, as can be seen at the example shown in Table III.

TABLE III. Results for a waveform not used at the training

\begin{tabular}{|c|c|c|}
\hline $\begin{array}{c}\text { waveform } \\
\text { coefficients }\end{array}$ & network output & error (\%) \\
\hline 288.3829 & 288.3694 & 0.005 \\
\hline 134.4744 & 134.4891 & 0.01 \\
\hline
\end{tabular}

\section{B. Harmonic detection by distorted waveforms}

Next trainings and simulations till the end of this work were carried out with unitary wave amplitudes for the fundamental harmonic, given that the use of higher values doesn't mean any difference for the ANN, because in these cases input normalization is done, with the consequence that the network operation is the same.

Now the MLP network is applied to the detection of the coefficients of the several harmonics present in a distorted waveform. For this purpose a proper set of training pairs are generated. They were distorted waves with different combinations of harmonics. The content in each harmonic was determined by the values of its rectangular components, making all possible combinations with either 0 or 0.2 as value for the different $A_{j} \mathrm{y} \mathrm{B}_{\mathrm{j}}$ of the several used harmonics. That is, either $0 \%$ or $20 \%$ of the amplitude of the fundamental wave. And that means that the amplitude of each harmonic oscillates between 0 and $28.28 \%$ and the phase angles go from -90 to 90 degrees.

With 5 harmonics the number of resulting distorted waveforms was 1024 . The required training times were relatively low (some minutes; always less than an hour in the PC above mentioned), and as results, a high accuracy could be reached. The mse reached with the training pairs by the training were of order $1 \mathrm{E}-8$. The needed number of neurons in these networks were $15+10$ neurons. And the errors at the outputs for distorted waveforms different of those of the training were clearly lower than $1 \%$ (of the order of $0.1 \%$ ).

Fig. 8 shows a waveform distorted with different amplitudes and phase angles for harmonics $3^{\circ}, 5^{\circ}, 7^{\circ}, 9^{\circ}$ and $11^{\circ}$. The training time with this particular harmonics was approx. 5 minutes. Table IV illustrates the results for the wave of Fig. 8. It shows the values for the coefficients corresponding to the rectangular components $A_{j} y B_{j}$ of these five harmonics. In one column the exact values of the waveform spectrum are shown, and next column contains the network outputs. In last column the resulting errors can be seen.

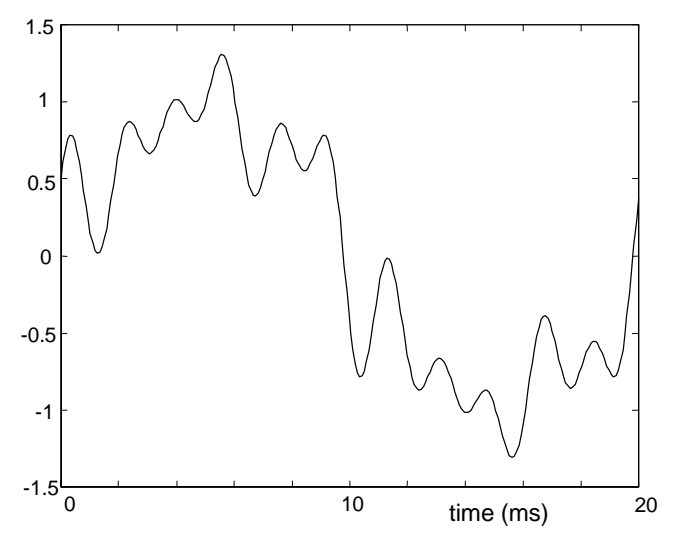

Fig. 8. Waveform with $3^{\text {rd }}, 5^{\text {th }}, 7^{\text {th }}, 9^{\text {th }}$ and $11^{\text {th }}$ harmonics

TABLE IV. Results MLP network

\begin{tabular}{|c|c|c|c|}
\hline coeffs. & $\begin{array}{c}\text { exact } \\
\text { values }\end{array}$ & $\begin{array}{c}\text { network } \\
\text { output }\end{array}$ & $\begin{array}{c}\text { error } \\
(\%)\end{array}$ \\
\hline A3 & 0 & 0.0000 & - \\
\hline B3 & 0.05 & 0.0501 & 0.2 \\
\hline A5 & 0.1 & 0.1001 & 0.1 \\
\hline B5 & 0.15 & 0.1502 & 0.13 \\
\hline A7 & 0.2 & 0.2000 & 0 \\
\hline B7 & 0 & -0.0002 & - \\
\hline A9 & 0.05 & 0.0499 & 0.2 \\
\hline B9 & 0.1 & 0.0998 & 0.2 \\
\hline A11 & 0.15 & 0.1501 & 0.07 \\
\hline B11 & 0.2 & 0.2003 & 0.15 \\
\hline
\end{tabular}

For higher number of harmonics the detection is possible too, although by means of an increasing number of neurons and increasing training times. In particular, this times grow exponentially with increasing number of coefficients. 


\section{Harmonic detection by using Elman Recurrent Network and Dynamic MLP}

First of all, these ANN topologies were tested for results by detecting harmonic amplitudes, then in case it works they could have certain advantages with respect to the static MLP, because of the way in which the inputs are introduced to the network. In other words, these networks have better dynamic features than the static MLP due to the more frequently updating of the output, with every new sample value from the signal that is introduced to the network, without having to wait for a set of values corresponding to a full period of the wave. Besides, the sequential introduction of the data is simple and more direct.

Nevertheless, the achieved results were of very low accuracy, compared with those of the static MLP. So the work with these two topologies centered on the second strategy above described.

\section{A. Approach to the problem and parameter adjustments}

At this second strategy it was tried to get as network output a fundamental frequency wave from a distorted waveform. Hence it seemed to be interesting to make an estimation of the basic parameters and ability of the network by obtaining a sinusoidal waveform in real time as output of the ANN when this same sinusoidal wave is introduced as input. So the training pairs consist of the same sinusoidal wave for both input and output of the neural network.

The best results with Elman network were obtained with 20 neurons in the hidden layer. In Fig. 9 it can be seen the result of a simulation after the training: two curves are represented, the sinusoidal input and the network output. The mean square error reached was mse $=6.5 \mathrm{E}-5$. It isn't a high accuracy, but can be considered acceptable.

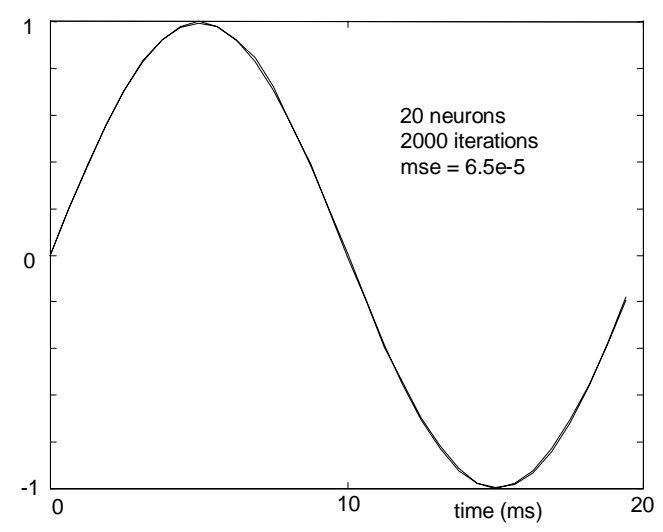

Fig. 9. Obtaining a sinusoidal waveform with the Elman network

But the generalization of the trained network to waves of different amplitudes with Elman network resulted to be very poor. To improve it trainings were carried out with two learning pairs of different amplitudes for getting better results in the range between both amplitudes. Waves of amplitudes 0.5 and 1.5 were used for this purpose, and the mean square errors obtained were all of the order of $1 \mathrm{E}-4$ for waves within this range. In other words, an improvement was achieved. But out of the range the accuracy was so lower as by the former trainings made.

By the application of the dynamic MLP, however, it was possible to generate the sinusoidal waveform at the network output with a very high accuracy (mse $=2.5 \mathrm{E}-11$ ), and, surprisingly, with a single neuron in the hidden layer, and in a training time of only 10 seconds. Fig. 10 contains both the input and the response of this dynamic MLP network, although it seems to be a single curve, then the output follows the input with a so low error. Besides, the network was able to generalize in a proper manner for amplitudes differents of that of the learning wave employed, including a wide range between 0 and 10. Fig. 11 shows the input and the output for the cases of amplitudes $0.5,0.7,0.9,1.1,1.3$ and 1.5. As in the case of introducing the learning waveform, it can't be appreciated the small distances between input and ouput curves.

With respect to the generalization for phase angle fluctuation, the accuracy was as high as with the learning wave. This could be expected for both Elman and dynamic MLP topologies, due to the sequential way of introducing the values and the "shortterm" memory of these topologies.

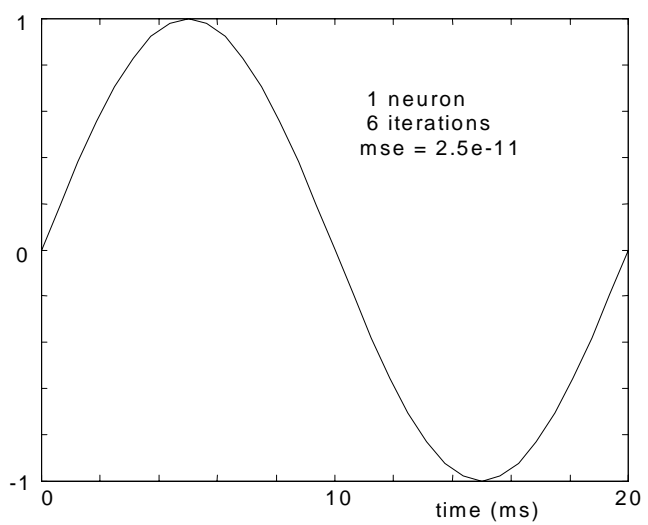

Fig. 10. Obtaining a sinusoidal waveform with the Dynamic MLP network

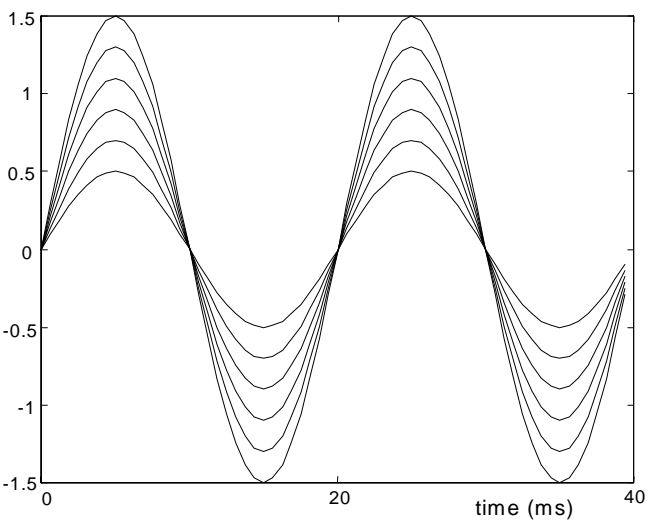

Fig. 11. Generalization for different amplitudes with the Dynamic MLP network 


\section{B. Extracting the instantaneous value of the fundamental wave from a waveform distorted by several harmonics}

Once known the parameters of both topologies for the previous case, it can be tried to extract the instantaneous value of the fundamental wave from a distorted waveform, not a pure sinusoidal one. The used training pairs were taken as the addition of a sinusoidal wave of amplitude 1 and combinations of 4 harmonics of amplitude 0.2 (15 different training waveforms). And as desired output a fundamental sinusoidal wave of amplitude 1 is employed.

The Elman network required $100+1$ neurons, while the dynamic MLP needed only 3+1 neurons and shorter training times. The obtained results with both topologies are shown in Table V. The first columns indicate the different training waveforms, specificating the harmonic content in each one. As can be seen at the other columns, where the mse for both ANNs appear as a result of simulating the network with these 15 waveforms, the dynamic MLP network has a much higher performance than the recurrent network. It is seen that this structure, with a secuentially introduced input and a set of delay units in the input layer confers the ANN a high performance, as could be proved too in other applications.[4],[8],[9].

TABLE V. Results Elman and Dynamic MLP networks

\begin{tabular}{|c|c|c|c|c|c|c|}
\hline wave & $5^{\circ}$ & $7^{\circ}$ & $11^{\circ}$ & $13^{\circ}$ & mse Elman & mse MLP Din. \\
\hline 1 & $\mathrm{x}$ & & & & 0.0019 & $2.4 \mathrm{e}-7$ \\
\hline 2 & & $\mathrm{x}$ & & & 0.0016 & $3.7 \mathrm{e}-7$ \\
\hline 3 & & & $\mathrm{x}$ & & 0.0021 & $4.6 \mathrm{e}-7$ \\
\hline 4 & & & & $\mathrm{x}$ & 0.0009 & $4.5 \mathrm{e}-7$ \\
\hline 5 & $\mathrm{x}$ & $\mathrm{x}$ & & & 0.0027 & $2.1 \mathrm{e}-7$ \\
\hline 6 & $\mathrm{x}$ & & $\mathrm{x}$ & & 0.0034 & $2.7 \mathrm{e}-7$ \\
\hline 7 & $\mathrm{x}$ & & & $\mathrm{x}$ & 0.0022 & $2.5 \mathrm{e}-7$ \\
\hline 8 & & $\mathrm{x}$ & $\mathrm{x}$ & & 0.0031 & $3.5 \mathrm{e}-7$ \\
\hline 9 & & $\mathrm{x}$ & & $\mathrm{x}$ & 0.0020 & $3.7 \mathrm{e}-7$ \\
\hline 10 & & & $\mathrm{x}$ & $\mathrm{x}$ & 0.0024 & $4.5 \mathrm{e}-7$ \\
\hline 11 & $\mathrm{x}$ & $\mathrm{x}$ & $\mathrm{x}$ & & 0.0043 & $2.1 \mathrm{e}-7$ \\
\hline 12 & $\mathrm{x}$ & $\mathrm{x}$ & & $\mathrm{x}$ & 0.0032 & $2.3 \mathrm{e}-7$ \\
\hline 13 & $\mathrm{x}$ & & $\mathrm{x}$ & $\mathrm{x}$ & 0.0036 & $2.7 \mathrm{e}-7$ \\
\hline 14 & & $\mathrm{x}$ & $\mathrm{x}$ & $\mathrm{x}$ & 0.0034 & $3.4 \mathrm{e}-7$ \\
\hline 15 & $\mathrm{x}$ & $\mathrm{x}$ & $\mathrm{x}$ & $\mathrm{x}$ & 0.0046 & $2.1 \mathrm{e}-7$ \\
\hline & & & & & \\
\hline
\end{tabular}

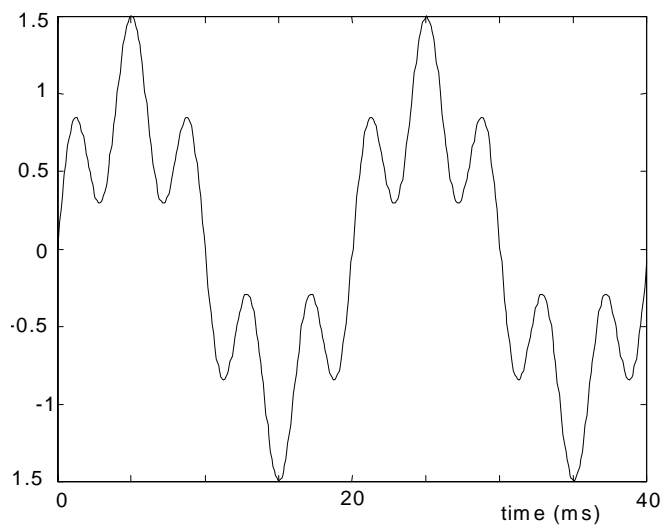

Fig. 12. Distorted waveform with a $5^{\text {th }}$ harmonic
In Fig. 12 a waveform highly distorted by the $5^{\text {th }}$ harmonic is presented, and Fig. 13 shows the fundamental wave of such a waveform and the output of the dynamic MLP. It can be observed how after the necessary time for the network to get enough information of the waveform, i. e. one complete cycle, the network ouput superposes the fundamental wave, meaning this fact that the mse is very low.

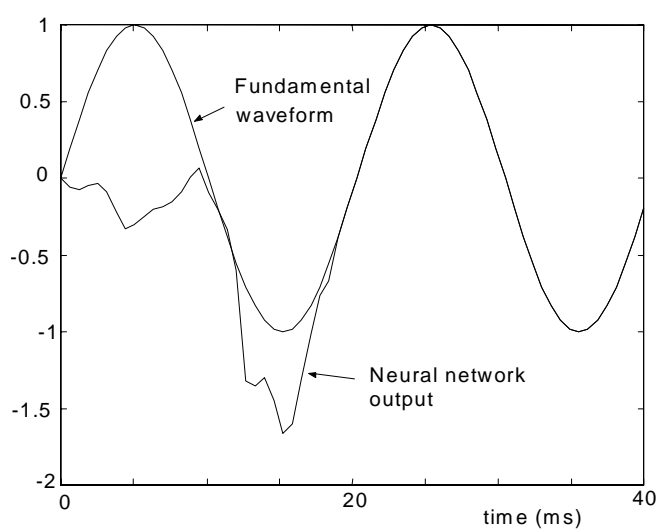

Fig. 13. Fundamental harmonic of waveform of Fig. 12 and output of the Dynamic MLP network

\section{Results discussion and conclusion}

The different neural networks are able to detect the harmonics present in voltage or current distorted waveforms. From the different architectures or types of networks, it were selected the most suitable ones depending on the detection strategy to be used for the APF or on the results obtained with them, by us and by other authors, in different types of applications.

By the strategy for detection of the harmonic spectrum, it is the MLP the one with the highest performance, getting to detect with enough accuracy, in short trainings (shorter than an hour in a personal computer) up to 5 or 6 harmonics, with a low number of neurons (from 8 to 15 for the hidden layer). In other works we could increase the number of harmonics detected into 10 , by using two MLP networks parallel-connected, each one of them detecting 5 different harmonics. This amounts of harmonics are in most of the practical cases a number enough to make able with the APF the elimination of the most distortion at the load. Besides, the network training can be adapted to the type of usual harmonic distortion at the load where they are going to be applied. Also the other two studied topologies have been applied to this first strategy trying to obtain the rectangular componentes of the harmonics, but only with low performance. They don't reach the minimum accuracy stablished as goal (1\% of relativ error).

The second strategy permits to extract the instantaneous value of the fundamental wave from a distorted waveform. The suitable neural networks are those with dynamic features, in other words, the ones with "shortterm memory". In particular the dynamic MLP showed its ability to obtain the fundamental wave in real 
time with a high accuracy, requiring for this a very low number of neurons. Only 3 neurons in the hidden layer were needed for waveforms distorted by 4 harmonics. The training times in this case were very short (a few minutes at a Pentium 1.7 GHz Personal Computer). On the other hand, the Elman recurrent network requires a very high number of neurons (aprox. 100 neurons for the mentioned task), reaching accuracy levels much lower than those of the dynamic MLP. And the training and operating times are longer because of the high number of neurons contained in the network. Thus, we consider this ANN topology not appropiate for application to the active power filters.

In short, from the comparative analysis made between different topologies of neural networks, it can be concluded that:

1) The static MLP network is the most appropiate for the first of the strategies.

2) The dynamic MLP network is the suitable topology for the second control strategy.

Both types of configurations or working methods result appropiate for determining of the reference signal at the APF control, were the second of them could have advantages in terms of higher application simplicity, due to the sequential data input, and the real time response of the network.

\section{References}

[1] N. Mohan, T. Undeland, W. Robbins, "Power electronics: converters, applications, and design", Wiley, 1995.

[2] P.K. Simpson, "Foundations of neural networks", Neural Networks Theory, Technology and Applications, 1993.

[3] C.V. Regueiro, S. Barro, E. Sánchez, M. FernándezDelgado, "Modelos básicos de redes neuronales artificiales", Computación Neuronal, Universidad de Santiago de Compostela. pp 181-218, 1995.

[4] T. Fukuda, T. Shibata, "Theory and Applications of Neural Networks for Industrial Control Systems", IEEE Transactions on Industrial Electronics, Vol. 39, No. 6, pp 472-489, December 1992.

[5] S.D. Round, N. Mohan, "Comparison of frequency and time domain neural network controllers for an active power filter", Proceedings of the IECON'93, IEEE International Conference on Industrial Electronics, Control and Instrumentation, Vol. 2, pp 1099-1104, 1993.

[6] N. Pecharanin, M. Sone, H. Mitsui, "An application of neural network for harmonic detection in active filter", IEEE World Congress on Computational Intelligence., IEEE International Conference on Neural Networks, Vol. 6, pp. 3756-3760, 1994.

[7] Y. Wada, N. Pecharanin, A. Taguchi, N. Iijima, Y. Akima, M. Sone, "Application of recurrent neural network for active filter", IEEE International Conference on Neural Networks, Proceedings, Vol.1, pp 488-491, 1995.

[8] H. Mori, K. Itou, H. Uematsu, S. Tsuzuki, "An artificial neural-net based method for predicting power system voltage harmonics", IEEE Transactions on Power Delivery, Vol 7, No. 1, pp 402-409, January 1992.

[9] S. Haykin, "Lessons on adaptative systems for signal processing, communications, and control”, IEEE Signal Processing Magazine, pp 39-48, September 1999

[10] M. Kim, G. Simoes, B.K. Bose, "Neural network-based estimation of power electronic waveforms", IEEE Transactions on Power Electronics, Vol 11, No. 2, pp 383-389, Marz 1996. 